\title{
Weakly almost periodic functionals on the measure algebra
}

\author{
Matthew Daws
}

July 5, 2018

\begin{abstract}
It is shown that the collection of weakly almost periodic functionals on the convolution algebra of a commutative Hopf von Neumann algebra is a $\mathrm{C}^{*}$-algebra. This implies that the weakly almost periodic functionals on $M(G)$, the measure algebra of a locally compact group $G$, is a $\mathrm{C}^{*}$-subalgebra of $M(G)^{*}=C_{0}(G)^{* *}$. The proof builds upon a factorisation result, due to Young and Kaiser, for weakly compact module maps. The main technique is to adapt some of the theory of corepresentations to the setting of general reflexive Banach spaces.

Subject classification: 43A10, 46L89 (Primary); 43A20, 43A60, 81R50 (Secondary).
\end{abstract}

\section{Introduction}

For a topological (semi-)group $G$, the space of weakly almost periodic functions on $G$ is the subspace of $C(G)$ consisting of those $f \in C(G)$ such that the (left) translates of $f$ form a relatively weakly-compact subset of $C(G)$. We denote this set by $\operatorname{WAP}(G)$. Then $\operatorname{WAP}(G)$ is a unital $\mathrm{C}^{*}$-subalgebra of $C(G)$, say with character space $G^{\text {WAP }}$. By continuity, we can extend the product from $G$ to $G^{\mathrm{WAP}}$, turning $G^{\mathrm{WAP}}$ into a compact semigroup whose product is separately continuous, a semitopological semigroup. Indeed, $G^{\mathrm{WAP}}$ is the universal semitopological semigroup compactification of $G$. See [2] or [11] for further details.

Now suppose that $G$ is a locally compact group, so we may form the Banach space $L^{1}(G)$, which becomes a Banach algebra with the convolution product. Then $L^{\infty}(G)$, as the dual of $L^{1}(G)$, naturally becomes an $L^{1}(G)$-bimodule. We define the space of weakly almost periodic functionals on $L^{1}(G)$, denoted by $\operatorname{WAP}\left(L^{1}(G)\right)$, to be the collection of $f \in L^{1}(G)$ such that the map

$$
R_{f}: L^{1}(G) \rightarrow L^{\infty}(G), \quad a \mapsto a \cdot f \quad\left(a \in L^{1}(G)\right),
$$

is weakly-compact. This is equivalent to the map $L_{f}: L^{1}(G) \rightarrow L^{\infty}(G), a \mapsto f \cdot a$ being weakly-compact. Ülger showed in $\left[20\right.$ that $\operatorname{WAP}\left(L^{1}(G)\right)=\operatorname{WAP}(G)$, where $C(G)$ is naturally identified with a subspace of $L^{\infty}(G)$. This fact also follows easily from [22, Lemma 6.3], using the fact that if a set is relatively weakly compact, then the weak- and weak*-topology closures coincide. Both these papers use simple bounded approximate identity arguments.

The definition of $\operatorname{WAP}\left(L^{1}(G)\right)$ obviously generalises to any Banach algebra $\mathcal{A}$. In general, we can say little about $\operatorname{WAP}(\mathcal{A})$, except for some interesting links with the Arens products, see [6] and references therein, or [17, Section 1.4] and [3, Theorem 2.6.15]. However, motivated by the above example, we might expect that when $\mathcal{A}$ has a large amount of structure, $\operatorname{WAP}(\mathcal{A})$ also might have extra structure. In this paper, we shall investigate $\operatorname{WAP}(M(G))$, where $M(G)$ is the measure algebra over a locally compact group $G$. In particular, we shall show that $\operatorname{WAP}(M(G))$ is a $\mathrm{C}^{*}$-subalgebra of $M(G)^{*}$, where $M(G)$ is identified as the dual of $C_{0}(G)$, so that $M(G)^{*}=C_{0}(G)^{* *}$ is a commutative von Neumann algebra. 
The central idea is to develop a theory of corepresentations on reflexive Banach spaces, for commutative Hopf von Neumann algebras. Our theory exactly replicates that for Hilbert spaces, but care needs to be taken to ensure that everything works in our more general setting.

The connection between weakly almost periodic functionals and representations of Banach algebras goes back to Young, [21], and Kaiser, [12]. For $L^{1}(G)$, there is a correspondence between (non-degenerate) representations of $L^{1}(G)$ and representations of $G$. Using Young and Kaiser's work, it is easy to see that weakly almost periodic functionals on $L^{1}(G)$ correspond to coefficient functionals for representations of $G$ on reflexive spaces. Then multiplication of functions in $L^{\infty}(G)$ corresponds to tensoring representations. The existence of reflexive tensor products (see [1] for example) hence shows that the product of two weakly almost periodic functionals is again weakly almost periodic. Of course, for $L^{1}(G)$, it is far easier to use Ülger's result, and then argue directly that $\operatorname{WAP}(G)$ is an algebra (which follows from Grothendieck's criteria for weak compactness, see [2]). For $M(G)$, while $M(G)=L^{1}(X)$ for some measure space $X$, we do not have that $X$ is a (semi)group, and so we turn to corepresentations, which work with the algebra $M(G)^{*}$ directly.

The structure of the paper is as follows. We first introduce some notions from the theory of tensor products of Banach spaces, in particular the projective and injective tensor norms. We then define what a (commutative) Hopf von Neumann algebra is, and show carefully that $M(G)$ (as well as $L^{1}(G)$ ) fits into this abstract framework. For the rest of the paper, we work with commutative Hopf von Neumann algebras, the results for $M(G)$ (and, indeed, $L^{1}(G)$ ) being immediate corollaries. As an immediate application, we make a quick study of almost periodic functionals. We then turn our attention to weakly almost periodic functionals, and build a theory of corepresentations on reflexive Banach spaces. The final application is then obtained by checking that the usual way of tensoring corepresentations still works in this more general setting.

For an introduction to quantum groups from a functional analysis viewpoint, [13], or the pair of articles [14] and [15], are very readable. A good starting point for details about (weakly) almost periodic functionals on general Banach algebras is [9].

A few notes on notion. We generally follow 3 for details about Banach algebras. We write $E^{*}$ for the dual of a Banach space $E$, and use the dual pairing notation $\langle\mu, x\rangle=\mu(x)$, for $\mu \in E^{*}$ and $x \in E$. We write $\mathcal{B}(E, F)$ for the collection of bounded linear maps from $E$ to $F$, we write $\mathcal{B}(E, E)=\mathcal{B}(E)$, and we write $T^{*}$ for the linear adjoint of an operator $T$.

Acknowledgements: The author would like to thank Garth Dales, for bringing this problem to his attention, and for careful proofreading. Thanks to Tony Lau for providing the reference [22].

\section{Hopf von Neumann algebras}

We start by recalling some elementary definitions and facts from the theory of tensor products of Banach spaces. We refer the reader to the books [18] and [7], or [8, Chapter VIII], for further details.

Let $E$ and $F$ be Banach spaces. The projective tensor norm, $\|\cdot\|_{\pi}$, on $E \otimes F$ is defined 
by

$$
\|\tau\|_{\pi}=\inf \left\{\sum_{k=1}^{n}\left\|x_{k}\right\|\left\|y_{k}\right\|: \tau=\sum_{k=1}^{n} x_{k} \otimes y_{k}\right\} \quad(\tau \in E \otimes F) .
$$

Then $E \widehat{\otimes} F$, the projective tensor product of $E$ and $F$, is the completion of $E \otimes F$ with respect to $\|\cdot\|_{\pi}$. The projective tensor product has the property that any bounded, bilinear map $\psi: E \times F \rightarrow G$ admits a unique bounded linear extension $\tilde{\psi}: E \widehat{\otimes} F \rightarrow G$, with $\|\tilde{\psi}\|=\|\psi\|$. For measure spaces $X$ and $Y$, we have that $L^{1}(X) \widehat{\otimes} L^{1}(Y)=L^{1}(X \times Y)$. We identify $(E \widehat{\otimes} F)^{*}$ with $\mathcal{B}\left(E, F^{*}\right)$ under the dual pairing

$$
\langle T, x \otimes y\rangle=\langle T(x), y\rangle \quad\left(T \in \mathcal{B}\left(E, F^{*}\right), x \in E, y \in F\right),
$$

and using linearity and continuity.

The injective tensor norm, $\|\cdot\|_{\epsilon}$, on $E \otimes F$ is defined by regarding $E \otimes F$ as a subspace of $\mathcal{B}\left(E^{*}, F\right)$, where $\tau=\sum_{k=1}^{n} x_{k} \otimes y_{k}$ induces the finite-rank operator

$$
E^{*} \rightarrow F, \quad \mu \mapsto \sum_{k=1}^{n}\left\langle\mu, x_{k}\right\rangle y_{k}
$$

Then $E \check{\otimes} F$, the injective tensor product of $E$ and $F$, is the completion of $E \otimes F$ with respect to $\|\cdot\|_{\epsilon}$. For locally compact Hausdorff spaces $K$ and $L$, we have that $C_{0}(K) \check{\otimes} C_{0}(L)=$ $C_{0}(K \times L)$. We write $\mathcal{A}(E, F)$ for the closure of the finite-rank operators from $E$ to $F$; these are the approximable operators from $E$ to $F$. Then, almost by definition, we have that $\mathcal{A}(E, F)=E^{*} \check{\otimes} F$.

There is a canonical norm-decreasing map $E \widehat{\otimes} F \rightarrow E \check{\otimes} F$. By taking the adjoint, we get an injective contraction $(E \check{\otimes} F)^{*} \rightarrow \mathcal{B}\left(E, F^{*}\right)$. The image, equipped with the norm induced by $(E \check{\otimes} F)^{*}$, is the space of integral operators, $\mathcal{I}\left(E, F^{*}\right)$. The map $E^{*} \widehat{\otimes} E \rightarrow E^{*} \check{\otimes} E$ is injective if and only if $E$ has the approximation property. We can regard $E^{*} \widehat{\otimes} E$ as a subspace of $\mathcal{I}\left(E^{*}\right)=\left(E^{*} \check{\otimes} E\right)^{*}=\mathcal{A}(E)^{*}$ by

$$
\langle\mu \otimes x, T\rangle=\langle\mu, T(x)\rangle \quad\left(T \in \mathcal{A}(E), \mu \otimes x \in E^{*} \widehat{\otimes} E\right) .
$$

Similarly, we can regard $E \widehat{\otimes} F$ as a subspace of $\mathcal{I}\left(E^{*}, F\right)$; here we use that fact that $\mathcal{I}\left(E^{*}, F\right)$ is isometrically a subspace of $\mathcal{I}\left(E^{*}, F^{* *}\right)=\left(E^{*} \check{\otimes} F^{*}\right)^{*}=\mathcal{A}\left(E, F^{*}\right)^{*}$. We say that $E$ has the metric approximation property if and only if the map $E^{*} \widehat{\otimes} E \rightarrow \mathcal{I}\left(E^{*}\right)$ is an isometry onto its range, or equivalent, $E \widehat{\otimes} F \rightarrow \mathcal{I}\left(E^{*}, F\right)$ is an isometry onto its range, for all $F$. There are characterisations of the (metric) approximation property in terms of finite-rank approximations of the identity on compact sets. We have that $C_{0}(K)$ and $L^{1}(X)$ have the metric approximation property for all $K$ and $X$.

\subsection{Commutative Hopf von Neumann algebras}

A Hopf von Neumann algebra is a von Neumann algebra $\mathcal{M}$ equipped with a coproduct $\Delta: \mathcal{M} \rightarrow \mathcal{M} \bar{\otimes} \mathcal{M}$. Here $\bar{\otimes}$ denotes the von Neumann tensor product. This means that $\Delta$ is a normal $*$-homomorphism, and that $(\Delta \otimes \mathrm{id}) \Delta=(\mathrm{id} \otimes \Delta) \Delta$, that is, $\Delta$ is coassociative.

We shall concentrate on the case where $\mathcal{M}$ is commutative, so that $\mathcal{M}=L^{\infty}(X)$ for some measure space $X$. Then $\mathcal{M} \bar{\otimes} \mathcal{M}=L^{\infty}(X \times X)$, and so, as $\Delta$ is normal, it drops to give a contractive map $\Delta_{*}: L^{1}(X \times X)=L^{1}(X) \widehat{\otimes} L^{1}(X) \rightarrow L^{1}(X)$. Hence $\Delta_{*}$ induces a 
contractive bilinear map $L^{1}(X) \times L^{1}(X) \rightarrow L^{1}(X)$. Then $\Delta$ being coassociative is equivalent to $\Delta_{*}$ being associative.

As both $\mathcal{M}$ and $\mathcal{M}_{*}$ are Banach algebras, we have natural module actions of $\mathcal{M}$ on $\mathcal{M}_{*}$ and of $\mathcal{M}_{*}$ on $\mathcal{M}$. For the action of $\mathcal{M}$ on $\mathcal{M}_{*}$, we shall, for example, write $F \cdot a \in \mathcal{M}_{*}$ for $F \in \mathcal{M}$ and $a \in \mathcal{M}_{*}$. For the action of $\mathcal{M}_{*}$ on $\mathcal{M}$, we shall always explicitly invoke the map $\Delta_{*}$ or $\Delta$.

For an example of a commutative Hopf von Neumann algebra, let $G$ be a locally compact group, and consider the algebra $L^{\infty}(G)$ equipped with the coproduct $\Delta$ defined by

$$
\Delta(f)(s, t)=f(s t) \quad\left(f \in L^{\infty}(G), s, t \in G\right) .
$$

Then $\Delta_{*}$ induces the usual convolution product on $L^{1}(G)$.

A slightly less well-known example is furnished by $M(G)$. As $M(G)=C_{0}(G)^{*}$, we see that $M(G)$ is the predual of the commutative von Neumann algebra $C_{0}(G)^{* *}$. As such, $M(G)^{*}=L^{\infty}(X)$ for some measure space $X$ (see [19, Chapter III]), and so by the uniqueness of preduals, $M(G)=L^{1}(X)$. Let $\Phi$ be the canonical coproduct on $C_{0}(G)$, so that $\Phi$ is the *-homomorphism $C_{0}(G) \rightarrow C(G \times G)$ defined by

$$
\Phi(f)(s, t)=f(s t) \quad\left(f \in C_{0}(G), s, t \in G\right) .
$$

We identify $C(G \times G)$, the space of bounded continuous functions on $G \times G$, with the multiplier algebra of $C_{0}(G \times G)$, and hence (see [19, Chapter III, Section 6]) we may identify $C(G \times G)$ with

$$
\left\{x \in C_{0}(G \times G)^{* *}: f x, x f \in C_{0}(G \times G)\left(f \in C_{0}(G \times G)\right)\right\} .
$$

We can hence regard $\Phi$ as a $*$-homomorphism $C_{0}(G) \rightarrow C_{0}(G \times G)^{* *}$.

We claim that $M(G) \widehat{\otimes} M(G)$ is, isometrically, a subspace of $M(G \times G)=C_{0}(G \times G)^{*}$. From the above, we can identify $M(G \times G)$ with $\mathcal{I}\left(C_{0}(G), M(G)\right)$. As $M(G)$ has the metric approximation property, we see that $M(G) \widehat{\otimes} M(G)$ is isometrically a subspace of $\mathcal{I}\left(M(G)^{*}, M(G)\right)$, or equivalently, by properties of the integral operators, isometrically a subspace of $\mathcal{I}\left(C_{0}(G), M(G)\right)$, as required.

Alternatively, for any $\mathrm{C}^{*}$-algebra $\mathcal{A}$, we could define a norm on $\mathcal{A}^{*} \otimes \mathcal{A}^{*}$ by embedding $\mathcal{A}^{*} \otimes \mathcal{A}^{*}$ into $\left(\mathcal{A} \otimes_{\min } \mathcal{A}\right)^{*}$. This induces the operator space projective tensor norm, see [10, Chapter 7], and as $\mathcal{A}$ has the minimal operator space structure, it follows that $\mathcal{A}^{*}$ has the maximal structure, and so this norm agrees with the (Banach space) projective tensor norm.

Hence $L^{\infty}(X) \bar{\otimes} L^{\infty}(X)=(M(G) \widehat{\otimes} M(G))^{*}$ is a quotient of $M(G \times G)^{*}=C_{0}(G \times G)^{* *}$. We claim that this quotient map is a $*$-homomorphism, for which it suffices to check that the kernel

$$
\left\{\tau \in C_{0}(G \times G)^{* *}:\langle\tau, \mu \otimes \lambda\rangle=0(\mu, \lambda \in M(G))\right\}
$$

is an ideal. Let $\mu, \lambda \in M(G)$, let $g \in C_{0}(G \times G)$, and let $f=f_{1} \otimes f_{2} \in C_{0}(G) \otimes C_{0}(G)$. Then

$$
\langle(\mu \otimes \lambda) \cdot f, g\rangle=\int f_{1}(s) f_{2}(t) g(s, t) d \mu(s) d \lambda(t)
$$

so $(\mu \otimes \lambda) \cdot f$ is the measure $\mu \cdot f_{1} \otimes \lambda \cdot f_{2} \in M(G) \otimes M(G)$. By continuity, we see that $(\mu \otimes \lambda) \cdot f \in M(G) \widehat{\otimes} M(G)$ for any $f \in C_{0}(G \times G)=C_{0}(G) \check{\otimes} C_{0}(G)$. Let $\tau$ be in the kernel, so that

$$
\langle\tau \cdot(\mu \otimes \lambda), f\rangle=\langle\tau,(\mu \otimes \lambda) \cdot f\rangle=0,
$$


as $\tau$ kills $M(G) \widehat{\otimes} M(G)$. Thus $\tau \cdot(\mu \otimes \lambda)=0$ in $C_{0}(G \times G)^{*}$. So, for $\sigma \in C_{0}(G \times G)^{* *}$, we see that

$$
\langle\sigma \tau, \mu \otimes \lambda\rangle=\langle\sigma, \tau \cdot(\mu \otimes \lambda)\rangle=0
$$

so that $\sigma \tau$ lies in the kernel.

Hence we have the following chain of $*$-homomorphisms

$$
C_{0}(G) \stackrel{\Phi}{\longrightarrow} C_{0}(G \times G)^{* *} \longrightarrow L^{\infty}(X \times X)=C_{0}(G)^{* *} \bar{\otimes} C_{0}(G)^{* *}
$$

say, giving rise to a $*$-homomorphism $\Delta_{0}: C_{0}(G) \rightarrow L^{\infty}(X \times X)$. There is hence a canonical extension (compare [19, Chapter III, Lemma 2.2]) $\Delta: L^{\infty}(X) \rightarrow L^{\infty}(X \times X)$, which is a normal $*$-homomorphism. Indeed, the preadjoint $\Delta_{*}: M(G) \widehat{\otimes} M(G) \rightarrow M(G)$ is defined by the chain of maps

$$
M(G) \widehat{\otimes} M(G)=L^{1}(X \times X) \longrightarrow L^{\infty}(X \times X)^{*} \stackrel{\Delta_{0}^{*}}{\longrightarrow} C_{0}(G)^{*}=M(G)
$$

Then, for $\mu, \lambda \in M(G)$ and $f \in C_{0}(G)$, we see that

$$
\left\langle\Delta_{*}(\mu \otimes \lambda), f\right\rangle=\left\langle\Delta_{0}(f), \mu \otimes \lambda\right\rangle=\int_{G \times G} f(s t) d \mu(s) d \lambda(t),
$$

so $\Delta_{*}$ induces the usual convolution product on $M(G)$. We have hence shown that $M(G)^{*}$ is a commutative Hopf von Neumann algebra. Notice that throughout, we have actually only used the fact that $G$ is a locally compact semigroup.

For a recent survey on measure algebras, see [4], where the authors view $M(G)$ as a $L a u$ algebra (see [16]).

\subsection{Almost periodic functionals}

For a Banach algebra $\mathcal{A}$, a functional $\mu \in \mathcal{A}^{*}$ is almost periodic if the map

$$
R_{\mu}: \mathcal{A} \rightarrow \mathcal{A}^{*}, \quad a \mapsto a \cdot \mu \quad(a \in \mathcal{A})
$$

is compact. We denote the collection of almost periodic functionals by $\operatorname{AP}(\mathcal{A})$. Then it is easy to see that $\operatorname{AP}(\mathcal{A})$ is a closed subspace of $\mathcal{A}^{*}$. Using the viewpoint of Hopf von Neumann algebras, it is easy to see that $\operatorname{AP}(M(G))$ is a $\mathrm{C}^{*}$-algebra.

Theorem 2.1. Let $\left(L^{\infty}(X), \Delta\right)$ be a commutative Hopf von Neumann algebra, so that $L^{1}(X)$ becomes a Banach algebra. Then $\operatorname{AP}\left(L^{1}(X)\right)$ is a $C^{*}$-subalgebra of $L^{\infty}(X)$.

Proof. Let $F \in \operatorname{AP}\left(L^{1}(X)\right) \subseteq L^{\infty}(X)$. For $f \in L^{1}(X)$, we shall write $f^{*}$ for the pointwise complex-conjugation of $f$, so that $f \mapsto f^{*}$ is the preadjoint of the involution on $L^{\infty}(X)$. We see that for $f, g \in L^{1}(X)$,

$$
\begin{aligned}
\left\langle R_{F^{*}}(f), g\right\rangle & =\left\langle F^{*}, \Delta_{*}(g \otimes f)\right\rangle=\left\langle\Delta(F)^{*}, g \otimes f\right\rangle=\left\langle F, \Delta_{*}\left(g^{*} \otimes f^{*}\right)\right\rangle \\
& =\left\langle R_{F}\left(f^{*}\right), g^{*}\right\rangle=\left\langle R_{F}\left(f^{*}\right)^{*}, g\right\rangle,
\end{aligned}
$$

so we conclude that $R_{F^{*}}$ is compact if and only if $R_{F}$ is compact. Hence $\operatorname{AP}\left(L^{1}(X)\right)$ is *-closed. 
We claim that $R_{F}=\Delta(F)^{*} \kappa_{L^{1}(X)}$. Indeed, for $f, g \in L^{1}(X)$, we have that

$$
\begin{aligned}
\left\langle R_{F}, f \otimes g\right\rangle & =\left\langle R_{F}(f), g\right\rangle=\left\langle F, \Delta_{*}(g \otimes f)\right\rangle=\langle\Delta(F), g \otimes f\rangle \\
& =\langle\Delta(F)(g), f\rangle=\left\langle\Delta(F) \kappa_{L^{1}(X)}(f), g\right\rangle .
\end{aligned}
$$

So $\Delta(F)=R_{F}^{*} \kappa_{L^{1}(X)}$, and hence $R_{F}$ is compact if and only if $\Delta(F)$ is compact. As $L^{\infty}(X)$ has the approximation property, it follows that $\Delta(F)$ is compact if and only if

$$
\Delta(F) \in \mathcal{A}\left(L^{1}(X), L^{\infty}(X)\right)=L^{\infty}(X) \check{\otimes} L^{\infty}(X)=L^{\infty}(X) \otimes_{\min } L^{\infty}(X) \subseteq L^{\infty}(X) \bar{\otimes} L^{\infty}(X) .
$$

Thus, if $F, G \in \operatorname{AP}\left(L^{1}(X)\right)$, then $\Delta(F), \Delta(G) \in L^{\infty}(X) \otimes_{\min } L^{\infty}(X)$, and so $\Delta(F G)=$ $\Delta(F) \Delta(G) \in L^{\infty}(X) \otimes_{\min } L^{\infty}(X)$, as $L^{\infty}(X) \otimes_{\min } L^{\infty}(X)$ is an algebra. Hence $F G \in$ $\mathrm{AP}\left(L^{1}(X)\right)$, as required.

Corollary 2.2. For a locally compact group $G, \operatorname{AP}(M(G))$ is a $C^{*}$-subalgebra of $M(G)^{*}$.

\section{Weakly almost periodic functionals}

We shall make use of vector valued $L^{p}$ spaces; for a measure space $X$, a Banach space $E$, and $1 \leq p<\infty$, we write $L^{p}(X, E)$ for the space of (classes of almost everywhere equal) Bochner $p$-integrable functions from $X$ to $E$. Then $L^{p}(X) \otimes E$ naturally maps into $L^{p}(X, E)$ with dense range, inducing a norm $\Delta_{p}$ on $L^{p}(X) \otimes E$. This norm is studied in [7, Chapter 7]. We have that $L^{1}(X) \widehat{\otimes} E=L^{1}(X, E)$, so that $\Delta_{1}=\|\cdot\|_{\pi}$, the projective tensor norm.

It is worth noting that $\Delta_{p}$ is not a tensor norm, as $T \in \mathcal{B}\left(L^{p}(X)\right)$ may fail to extend to a bounded map $T \otimes$ id $: L^{p}(X, E) \rightarrow L^{p}(X, E)$. However, note that for $F \in L^{\infty}(X)$, then denoting also by $F$ the multiplication operator on $L^{p}(X)$, it is elementary that $F \otimes$ id is bounded, with norm $\|F\|$, on $L^{p}(X, E)$. The norm $\Delta_{p}$ does satisfy the estimates

$$
\|\tau\|_{\epsilon} \leq \Delta_{p}(\tau) \leq\|\tau\|_{\pi} \quad\left(\tau \in L^{p}(X) \otimes E\right),
$$

so in particular, $\Delta_{p}(f \otimes x)=\|f\|\|x\|$ for $f \in L^{p}(X)$ and $x \in E$.

We shall henceforth restrict to the case where $E$ is reflexive. Then $E^{*}$ has the RadonNikodým property, and so $L^{p}(X, E)^{*}=L^{p^{\prime}}\left(X, E^{*}\right)$ for $1<p<\infty$, where $1 / p^{\prime}=1-1 / p$, see [7, Appendix D], or [8], for further details. We stress that even when $p=2$, the dual pairing between $L^{2}(X, E)$ and $L^{2}\left(X, E^{*}\right)$ is always bilinear and not sesquilinear.

Lemma 3.1. Let $E$ be a reflexive Banach space, and let $X$ be a measure space. The map

$$
\Lambda:\left(L^{2}(X) \otimes E^{*}\right) \times\left(L^{2}(X) \otimes E\right) \rightarrow L^{1}(X) \otimes\left(E^{*} \widehat{\otimes} E\right) ;(f \otimes \mu, g \otimes x) \mapsto f g \otimes(\mu \otimes x)
$$

extends to a metric surjection

$$
\Lambda: L^{2}\left(X, E^{*}\right) \widehat{\otimes} L^{2}(X, E) \rightarrow L^{1}\left(X, E^{*} \widehat{\otimes} E\right)=L^{1}(X) \widehat{\otimes} E^{*} \widehat{\otimes} E .
$$

Here $f g$ denotes the pointwise product, so the Cauchy-Schwarz inequality shows that $f g \in$ $L^{1}(X)$ for $f, g \in L^{2}(X)$.

Proof. Let $F \in L^{2}\left(X, E^{*}\right)$ and $G \in L^{2}(X, E)$ be simple functions, so that there exists a disjoint partition of $X$, say $\left(X_{k}\right)_{k=1}^{n}$, and $\left(x_{k}\right)_{k=1}^{n} \subseteq E$ and $\left(\mu_{k}\right)_{k=1}^{n} \subseteq E^{*}$ with

$$
F=\sum_{k=1}^{n} \chi_{X_{k}} \otimes \mu_{k}, \quad G=\sum_{k=1}^{n} \chi_{X_{k}} \otimes x_{k} .
$$


Here we write $\chi_{X_{k}}$ for the indicator function of $X_{k}$. Hence we see that

$$
\Lambda(F \otimes G)=\sum_{k=1}^{n} \chi_{X_{k}} \otimes\left(\mu_{k} \otimes x_{k}\right)
$$

which has norm

$$
\sum_{k=1}^{n}\left|X_{k}\right|\left\|\mu_{k} \otimes x_{k}\right\| \leq\left(\sum_{k=1}^{n}\left|X_{k}\right|\left\|\mu_{k}\right\|^{2}\right)^{1 / 2}\left(\sum_{k=1}^{n}\left|X_{k}\right|\left\|x_{k}\right\|^{2}\right)^{1 / 2}=\|F\|\|G\| .
$$

As the simple functions are dense in $L^{2}(X, E)$, respectively, $L^{2}\left(X, E^{*}\right)$, we conclude that the map $\Lambda: L^{2}\left(X, E^{*}\right) \times L^{2}(X, E) \rightarrow L^{1}\left(X, E^{*} \widehat{\otimes} E\right)$ is a contraction, and so extends to a contraction $L^{2}\left(X, E^{*}\right) \widehat{\otimes} L^{2}(X, E) \rightarrow L^{1}\left(X, E^{*} \widehat{\otimes} E\right)$.

As $E$ is reflexive, we may identify $\left(E^{*} \widehat{\otimes} E\right)^{*}$ with $\mathcal{B}(E)$. Hence $\Lambda^{*}$ is a map $\mathcal{B}\left(L^{1}(X), \mathcal{B}(E)\right) \rightarrow$ $\mathcal{B}\left(L^{2}(X, E)\right)$, say $\pi \mapsto W$, where

$$
\langle f \otimes \mu, W(g \otimes x)\rangle=\langle\mu, \pi(f g)(x)\rangle \quad\left(f, g \in L^{2}(X), \mu \in E^{*}, x \in E\right) .
$$

By a suitable choice of $f, g, x$ and $\mu$, we see that $\|W\| \geq\|\pi\|$, and so we conclude that actually $\|W\|=\|\pi\|$. Hence $\Lambda^{*}$ is an isometry, so $\Lambda$ must be a metric surjection, as required.

For $F \in L^{\infty}(G)$ and $T \in \mathcal{B}(E)$, we see that $F \otimes T$ extends to a bounded linear map on $L^{2}(X, E)$. Let $L^{\infty}(X) \bar{\otimes} \mathcal{B}(E)$ be the weak*-closure of $L^{\infty}(X) \otimes \mathcal{B}(E)$ inside $\mathcal{B}\left(L^{2}(X, E)\right)$. This is then a dual Banach algebra, that is, multiplication in $L^{\infty}(X) \bar{\otimes} \mathcal{B}(E)$ is separately weak*-continuous. See [6, Section 8], where similar ideas are explored.

Proposition 3.2. The above lemma isometrically identifies $\mathcal{B}\left(L^{1}(X), \mathcal{B}(E)\right)$ with a subspace of $\mathcal{B}\left(L^{2}(X, E)\right)$, under the mapping $\Lambda^{*}$. The image of $\Lambda^{*}$ is precisely $L^{\infty}(X) \bar{\otimes} \mathcal{B}(E)$.

Proof. Standard Banach space theory shows that the image of $\Lambda^{*}$ is equal to

$$
(\operatorname{ker} \Lambda)^{\perp}=\left\{T \in \mathcal{B}\left(L^{2}(X, E)\right):\langle T, \tau\rangle=0\left(\tau \in L^{2}\left(X, E^{*}\right) \widehat{\otimes} L^{2}(X, E), \Lambda(\tau)=0\right)\right\} .
$$

Hence the image of $\Lambda^{*}$ is weak*-closed. Notice that $L^{\infty}(X) \bar{\otimes} \mathcal{B}(E)$ is equal to $Z^{\perp}$, where

$$
Z=\left\{\tau \in L^{2}\left(X, E^{*}\right) \widehat{\otimes} L^{2}(X, E):\langle F \otimes S, \tau\rangle=0\left(F \in L^{\infty}(X), S \in \mathcal{B}(E)\right)\right\} .
$$

Hence we need to show that $\operatorname{ker} \Lambda=Z$.

Let $F \in L^{\infty}(X)$ and $S \in \mathcal{B}(E)$. Then let $T=F \otimes S \in \mathcal{B}\left(L^{2}(X, E)\right)$, and let $\pi: L^{1}(X) \rightarrow$ $\mathcal{B}(E)$ be the rank-one operator induced by $F \otimes S$, that is, $\pi(a)=\langle F, a\rangle S$ for $a \in L^{1}(X)$. Then $\Lambda^{*}(\pi)=T$, from which it follows that $\operatorname{ker} \Lambda \subseteq Z$.

As $L^{1}(X)$ has the approximation property, for each non-zero $\sigma \in L^{1}(X) \widehat{\otimes}\left(E^{*} \widehat{\otimes} E\right)$, there exists $F \in L^{\infty}(X)$ and $S \in \mathcal{B}(E)$ with $\langle F \otimes S, \sigma\rangle \neq 0$. Hence, if $\tau \in L^{2}\left(X, E^{*}\right) \widehat{\otimes} L^{2}(X, E)$ is such that $\sigma=\Lambda(\tau) \neq 0$, then there exists $T \in L^{\infty}(X) \otimes \mathcal{B}(E)$ with $0 \neq\langle T, \sigma\rangle=\left\langle\Lambda^{*}(T), \tau\right\rangle$. This shows that $Z \subseteq \operatorname{ker} \Lambda$.

Informally, the above proposition allows us to write

$$
\mathcal{B}\left(L^{1}(X), \mathcal{B}(E)\right)=\left(L^{1}(X) \widehat{\otimes}\left(E^{*} \widehat{\otimes} E\right)\right)^{*}=L^{\infty}(X) \bar{\otimes} \mathcal{B}(E),
$$

which is reminiscent of the operator space projective tensor result that $\left(\mathcal{M}_{*} \widehat{\otimes} \mathcal{N}_{*}\right)^{*}=\mathcal{M} \bar{\otimes} \mathcal{N}$, for von Neumann algebras $\mathcal{M}$ and $\mathcal{N}$, see [10, Theorem 7.2.4]. The important point for us 
is that we have turned $\mathcal{B}\left(L^{1}(X), \mathcal{B}(E)\right)$ into an algebra. It is multiplication in this algebra which will ultimately give rise to the multiplication of weakly almost periodic functionals in $L^{\infty}(X)$.

Now let $L^{\infty}(X)$ be a Hopf von Neumann algebra, so it admits a coproduct $\Delta$. We have a map

$$
\Delta_{*} \otimes \mathrm{id}: L^{1}(X \times X) \widehat{\otimes}\left(E^{*} \widehat{\otimes} E\right) \rightarrow L^{1}(X) \widehat{\otimes}\left(E^{*} \widehat{\otimes} E\right),
$$

whose adjoint, which we denote by $\Delta \otimes \mathrm{id}$, is a map

$$
\Delta \otimes \mathrm{id}: L^{\infty}(X) \bar{\otimes} \mathcal{B}(E) \rightarrow L^{\infty}(X \times X) \bar{\otimes} \mathcal{B}(E),
$$

where, of course, $L^{\infty}(X \times X) \bar{\otimes} \mathcal{B}(E)$ is a subalgebra of $\mathcal{B}\left(L^{2}(X \times X, E)\right)$.

Lemma 3.3. With notation as above, $(\Delta \otimes \mathrm{id})$ is a homomorphism.

Proof. Let $F \in L^{\infty}(X)$ and $a, b \in L^{1}(X)$. As $\Delta$ is a homomorphism, it follows that

$$
\Delta_{*}(\Delta(F) \cdot(a \otimes b))=F \cdot \Delta_{*}(a \otimes b) .
$$

Let $U \in L^{\infty}(X) \bar{\otimes} \mathcal{B}(E)$, let $T \in \mathcal{B}(E)$, and let $V=F \otimes T$. For $\tau \in E^{*} \widehat{\otimes} E$, we see that

$$
\begin{aligned}
\langle(\Delta \otimes \mathrm{id})(U V), a \otimes b \otimes \tau\rangle & =\left\langle U(F \otimes T), \Delta_{*}(a \otimes b) \otimes \tau\right\rangle=\left\langle U, F \cdot \Delta_{*}(a \otimes b) \otimes T \cdot \tau\right\rangle \\
& =\left\langle U, \Delta_{*}(\Delta(F) \cdot(a \otimes b)) \otimes T \cdot \tau\right\rangle \\
& =\langle(\Delta \otimes \mathrm{id}) U, \Delta(F) \cdot(a \otimes b) \otimes T \cdot \tau\rangle \\
& =\langle((\Delta \otimes \mathrm{id}) U)((\Delta \otimes \mathrm{id}) V), a \otimes b \otimes \tau\rangle .
\end{aligned}
$$

By linearity, we conclude that $(\Delta \otimes \mathrm{id})(U V)=((\Delta \otimes \mathrm{id}) U)((\Delta \otimes \mathrm{id}) V)$ for all $U \in$ $L^{\infty}(X) \bar{\otimes} \mathcal{B}(E)$ and $V \in L^{\infty}(X) \otimes \mathcal{B}(E)$. By weak*-continuity, this must also hold for $V \in L^{\infty}(X) \bar{\otimes} \mathcal{B}(E)$.

We now wish to adapt leg numbering notation to our setup. Given $W \in \mathcal{B}\left(L^{2}(X, E)\right)$, define $W_{23} \in \mathcal{B}\left(L^{2}(X \times X, E)\right)$ by

$$
W_{23}\left(f_{1} \otimes f_{2} \otimes x\right)=f_{1} \otimes W\left(f_{2} \otimes x\right) \quad\left(f_{1}, f_{2} \in L^{2}(X), x \in E\right) .
$$

Using the fact that $L^{2}(X \times X, E)=L^{2}\left(X, L^{2}(X, E)\right)$, it is easy to see that $W \mapsto W_{23}$ is a weak*-continuous, isometric mapping. If $W=F \otimes S$ for some $F \in L^{\infty}(X)$ and $S \in \mathcal{B}(E)$, then clearly $W_{23}=1 \otimes F \otimes S \in L^{\infty}(X \times X) \otimes \mathcal{B}(E)$. By weak*-continuity, we conclude that if $W \in L^{\infty}(X) \bar{\otimes} \mathcal{B}(E)$, then $W_{23} \in L^{\infty}(X \times X) \bar{\otimes} \mathcal{B}(E)$.

Let $\chi: L^{2}(X \times X) \rightarrow L^{2}(X \times X)$ be the "swap map", defined on elementary tensors by $\chi(f \otimes g)=g \otimes f$. For $W \in L^{\infty}(X \times X) \bar{\otimes} \mathcal{B}(E)$, it is clear that $(\chi \otimes \mathrm{id}) W$ and $W(\chi \otimes \mathrm{id})$ both also lie in $L^{\infty}(X \times X) \bar{\otimes} \mathcal{B}(E)$. For $W \in L^{\infty}(X) \bar{\otimes} \mathcal{B}(E)$, we define $W_{13}=(\chi \otimes$ id $) W_{23}(\chi \otimes$ id $) \in$ $L^{\infty}(X \times X) \bar{\otimes} \mathcal{B}(E)$.

Theorem 3.4. Let $\left(L^{\infty}(X), \Delta\right)$ be a Hopf von Neumann algebra, and let $E$ be a reflexive Banach space. Let $\pi: L^{1}(X) \rightarrow \mathcal{B}(E)$ be a bounded linear map, giving rise to $W \in$ $L^{\infty}(X) \bar{\otimes} \mathcal{B}(E)$. Then $\pi$ is a homomorphism, with respect to $\Delta_{*}$, if and only if $(\Delta \otimes \mathrm{id}) W=$ $W_{13} W_{23}$. 
Proof. Let $f_{1}, f_{2}, g_{1}, g_{2} \in L^{2}(X), \mu \in E^{*}$ and $x \in E$. Then

$$
\begin{aligned}
\left\langle f_{1} \otimes f_{2} \otimes \mu,(\Delta \otimes \mathrm{id}) W\left(g_{1} \otimes g_{2} \otimes x\right)\right\rangle & =\left\langle\pi, \Delta_{*}\left(f_{1} g_{1} \otimes f_{2} g_{2}\right) \otimes(\mu \otimes x)\right\rangle \\
& =\left\langle\mu, \pi\left(\Delta_{*}\left(f_{1} g_{1} \otimes f_{2} g_{2}\right)\right)(x)\right\rangle .
\end{aligned}
$$

We now come to a proof where "Sweedler notation" would help greatly, but we should perhaps, at least once, give a formal proof. Informally, we shall "pretend" that $W\left(g_{2} \otimes x\right)=$ $h \otimes y$. Then

$$
\begin{aligned}
\left\langle\mu, \pi\left(f_{1} g_{1}\right) \pi\left(f_{2} g_{2}\right)(x)\right\rangle & =\left\langle\pi\left(f_{1} g_{1}\right)^{*}(\mu), \pi\left(f_{2} g_{2}\right)(x)\right\rangle=\left\langle f_{2} \otimes \pi\left(f_{1} g_{1}\right)^{*}(\mu), W\left(g_{2} \otimes x\right)\right\rangle \\
& =\left\langle f_{2}, h\right\rangle\left\langle\mu, \pi\left(f_{1} g_{1}\right)(y)\right\rangle=\left\langle f_{2}, h\right\rangle\left\langle f_{1} \otimes \mu, W\left(g_{1} \otimes y\right)\right\rangle \\
& =\left\langle f_{1} \otimes f_{2} \otimes \mu, W_{13}\left(g_{1} \otimes h \otimes y\right)\right\rangle \\
& =\left\langle f_{1} \otimes f_{2} \otimes \mu, W_{13} W_{23}\left(g_{1} \otimes g_{2} \otimes x\right)\right\rangle
\end{aligned}
$$

which completes the proof.

To make this rigorous, for $\epsilon>0$, we can find a finite sum of elementary tensors $\sum_{k} h_{k} \otimes$ $y_{k} \in L^{2}(X) \otimes E$ with $\left\|W\left(g_{2} \otimes x\right)-\sum_{k} h_{k} \otimes y_{k}\right\|<\epsilon$. Then

$$
\left\|\left\langle f_{2} \otimes \pi\left(f_{1} g_{1}\right)^{*}(\mu), W\left(g_{2} \otimes x\right)\right\rangle-\sum_{k}\left\langle f_{2}, h_{k}\right\rangle\left\langle\mu, \pi\left(f_{1} g_{1}\right)\left(y_{k}\right)\right\rangle\right\|<\epsilon\left\|f_{2}\right\|\|\pi\|\left\|f_{1}\right\|\left\|g_{1}\right\|\|\mu\|,
$$

and, as above,

$$
\sum_{k}\left\langle f_{2}, h_{k}\right\rangle\left\langle\mu, \pi\left(f_{1} g_{1}\right)\left(y_{k}\right)\right\rangle=\sum_{k}\left\langle f_{1} \otimes f_{2} \otimes \mu, W_{13}\left(g_{1} \otimes h_{k} \otimes y_{k}\right)\right\rangle,
$$

so approximating again,

$$
\begin{aligned}
& \left\|\sum_{k}\left\langle f_{1} \otimes f_{2} \otimes \mu, W_{13}\left(g_{1} \otimes h_{k} \otimes y_{k}\right)\right\rangle-\left\langle f_{1} \otimes f_{2} \otimes \mu, W_{13} W_{23}\left(g_{1} \otimes g_{2} \otimes x\right)\right\rangle\right\| \\
& \quad<\epsilon\|W\|\left\|f_{1}\right\|\left\|f_{2}\right\|\|\mu\|\left\|g_{1}\right\|,
\end{aligned}
$$

and so

$$
\begin{aligned}
\mid\left\langle\mu, \pi\left(f_{1} g_{1}\right) \pi\left(f_{2} g_{2}\right)(x)\right\rangle & -\left\langle f_{1} \otimes f_{2} \otimes \mu, W_{13} W_{23}\left(g_{1} \otimes g_{2} \otimes x\right)\right\rangle \mid \\
& <2 \epsilon\|W\|\left\|f_{1}\right\|\left\|f_{2}\right\|\|\mu\|\left\|g_{1}\right\| .
\end{aligned}
$$

As $\epsilon>0$ was arbitrary, the proof is complete.

\subsection{Application to weakly almost periodic elements}

The following result was first shown by Young in [21], building upon [5], and was recast in terms of the real interpolation method by Kaiser in [12] (see also the similar arguments in [6]).

Theorem 3.5. Let $\mathcal{A}$ be a Banach algebra, and let $\mu \in \mathcal{A}^{*}$. The following are equivalent:

1. $\mu \in \operatorname{WAP}(\mathcal{A})$;

2. there exists a reflexive Banach space $E$, a contractive homomorphism $\pi: \mathcal{A} \rightarrow \mathcal{B}(E)$, and $x \in E, \lambda \in E^{*}$ such that

$$
\langle\mu, a\rangle=\langle\lambda, \pi(a)(x)\rangle \quad(a \in \mathcal{A}) .
$$


We shall need a way of tensoring reflexive Banach spaces in a way that gives a reflexive Banach space. As we do not wish to get bogged down in the details of any one specific way to do this, so we shall make an ad hoc definition.

Definition 3.6. Let $E$ and $F$ be reflexive Banach spaces, and let $\alpha$ be some norm on $E \otimes F$ such that:

1. we have that $\|\tau\|_{\epsilon} \leq \alpha(\tau) \leq\|\tau\|_{\pi}$ for each $\tau \in E \otimes F$;

2. the completion of $E \otimes F$ with respect to $\alpha$ is a reflexive Banach space, say $E \widehat{\otimes}_{\alpha} F$;

3. given $T \in \mathcal{B}(E)$ and $S \in \mathcal{B}(F)$, the map $T \otimes S: E \otimes F \rightarrow E \otimes F$ extends to a bounded operator on $E \widehat{\otimes}_{\alpha} F$ with norm $\|T\|\|S\|$.

Then we say that $\|\cdot\|$ is a reflexive tensor norm on $E \otimes F$.

The existence of reflexive tensor norms is shown in [1], for example.

Let $E$ and $F$ be reflexive Banach spaces, and let $\alpha$ be a reflexive tensor norm on $E \otimes F$. As the map $E \widehat{\otimes} F \rightarrow E \widehat{\otimes}_{\alpha} F$ is contractive with dense range, the adjoint $\left(E \widehat{\otimes}_{\alpha} F\right)^{*} \rightarrow \mathcal{B}\left(E, F^{*}\right)$ is injective. We write $\mathcal{B}_{\alpha^{\prime}}\left(E, F^{*}\right)$ for the image, and equip it with the norm coming from $\left(E \widehat{\otimes}_{\alpha} F\right)^{*}$, so we may write $\left(E \widehat{\otimes}_{\alpha} F\right)^{*}=\mathcal{B}_{\alpha^{\prime}}\left(E, F^{*}\right)$. For some norms $\alpha$, there exists a dual norm $\alpha^{\prime}$, which is a reflexive tensor norm on $E^{*} \otimes F^{*}$, such that $\mathcal{B}_{\alpha^{\prime}}\left(E, F^{*}\right)=E^{*} \widehat{\otimes}_{\alpha^{\prime}} F^{*}$. We shall, however, not have to assume this extra condition. For us, it suffices to note that as $\alpha$ dominates $\|\cdot\|_{\epsilon}$, there is a natural embedding of $E^{*} \otimes F^{*}$ into $\left(E \widehat{\otimes}_{\alpha} F\right)^{*}$.

Theorem 3.7. Let $\left(L^{\infty}(X), \Delta\right)$ be a commutative Hopf von Neumann algebra, and use $\Delta_{*}$ to turn $L^{1}(X)$ into a Banach algebra. Then $\operatorname{WAP}\left(L^{1}(X)\right)$ is a $C^{*}$-subalgebra of $L^{\infty}(X)$.

Proof. We know that WAP $\left(L^{1}(X)\right)$ is a closed subspace of $L^{\infty}(X)$. Exactly the same argument as in the proof of Theorem 2.1 shows that $\operatorname{WAP}\left(L^{1}(X)\right)$ is $*$-closed, so it remains only to show that $\operatorname{WAP}\left(L^{1}(X)\right)$ is closed under multiplication.

Let $F_{1}, F_{2} \in \operatorname{WAP}\left(L^{1}(X)\right)$. By Theorem 3.5, for $i=1,2$, there exists a reflexive Banach space $E_{i}$, a contractive homomorphism $\pi_{i}: L^{1}(X) \rightarrow \mathcal{B}\left(E_{i}\right), x_{i} \in E_{i}$ and $\mu_{i} \in E_{i}^{*}$ such that

$$
\left\langle F_{i}, a\right\rangle=\left\langle\mu_{i}, \pi_{i}(a)\left(x_{i}\right)\right\rangle \quad\left(a \in L^{1}(X)\right) .
$$

Let $\alpha$ be a reflexive tensor norm on $E_{1} \otimes E_{2}$, and define $\hat{\pi}_{i}: L^{1}(X) \rightarrow \mathcal{B}\left(E_{1} \widehat{\otimes}_{\alpha} E_{2}\right)$, for $i=1,2$, by

$$
\hat{\pi}_{1}(a)=\pi_{1}(a) \otimes \mathrm{id}, \quad \hat{\pi}_{2}(a)=\mathrm{id} \otimes \pi_{2}(a) \quad\left(a \in L^{1}(X)\right) .
$$

Then $\hat{\pi}_{1}$ and $\hat{\pi}_{2}$ are contractive homomorphisms from $L^{1}(X)$ to $\mathcal{B}\left(E_{1} \widehat{\otimes}_{\alpha} E_{2}\right)$, and hence give rise, respectively, to $U, V \in L^{\infty}(X) \bar{\otimes} \mathcal{B}\left(E_{1} \widehat{\otimes}_{\alpha} E_{2}\right)$, such that

$$
(\Delta \otimes \mathrm{id}) U=U_{13} U_{23}, \quad(\Delta \otimes \mathrm{id}) V=V_{13} V_{23} .
$$

Let $W=U V \in L^{\infty}(X) \bar{\otimes} \mathcal{B}\left(E_{1} \widehat{\otimes}_{\alpha} E_{2}\right)$, and let $\pi: L^{1}(X) \rightarrow \mathcal{B}\left(E_{1} \widehat{\otimes}_{\alpha} E_{2}\right)$ be induced by $W$. By Lemma 3.3, we see that

$$
(\Delta \otimes \mathrm{id}) W=((\Delta \otimes \mathrm{id}) U)((\Delta \otimes \mathrm{id}) V)=U_{13} U_{23} V_{13} V_{23} .
$$

We also see that

$$
W_{13} W_{23}=U_{13} V_{13} U_{23} V_{23} .
$$


We claim that $U_{23} V_{13}=V_{13} U_{23}$, from which it follows, from Theorem 3.4, then $\pi$ is a homomorphism.

To prove the claim, we shall again deploy Sweedler notation: the argument can be made rigorous in the same way as in the proof of Theorem 3.4. Let $f_{1}, f_{2}, g_{1}, g_{2} \in L^{2}(X), w_{1} \in E_{1}$, $w_{2} \in E_{2}$ and $T \in \mathcal{B}_{\alpha^{\prime}}\left(E_{1}, E_{2}^{*}\right)=\left(E_{1} \widehat{\otimes}_{\alpha} E_{2}\right)^{*}$. Suppose that $U^{*}\left(f_{2} \otimes T\right)=h_{1} \otimes S_{1}$, so that for $k \in L^{2}(X), z_{1} \in E_{1}$ and $z_{2} \in E_{2}$, we have that

$$
\begin{aligned}
\left\langle h_{1} \otimes S_{1}, k \otimes z_{1} \otimes z_{2}\right\rangle & =\left\langle f_{2} \otimes T, U\left(k \otimes z_{1} \otimes z_{2}\right)\right\rangle=\left\langle T, \pi_{1}\left(f_{2} k\right)\left(z_{1}\right) \otimes z_{2}\right\rangle \\
& =\left\langle T \pi_{1}\left(f_{2} k\right)\left(z_{1}\right), z_{2}\right\rangle .
\end{aligned}
$$

Similarly, suppose that $V^{*}\left(f_{1} \otimes T\right)=h_{2} \otimes S_{2}$, so that

$$
\begin{aligned}
\left\langle h_{2} \otimes S_{2}, k \otimes z_{1} \otimes z_{2}\right\rangle & =\left\langle f_{1} \otimes T, V\left(k \otimes z_{1} \otimes z_{2}\right)\right\rangle=\left\langle T, z_{1} \otimes \pi_{2}\left(f_{1} k\right)\left(z_{2}\right)\right\rangle \\
& =\left\langle T\left(z_{1}\right), \pi_{2}\left(f_{1} k\right)\left(z_{2}\right)\right\rangle .
\end{aligned}
$$

Thus we see that

$$
\begin{aligned}
& \left\langle f_{1} \otimes f_{2} \otimes T, U_{23} V_{13}\left(g_{1} \otimes g_{2} \otimes w_{1} \otimes w_{2}\right)\right\rangle \\
& =\left\langle f_{1} \otimes U^{*}\left(f_{2} \otimes T\right),(\chi \otimes \mathrm{id} \otimes \mathrm{id})\left(g_{2} \otimes V\left(g_{1} \otimes w_{1} \otimes w_{2}\right)\right)\right\rangle \\
& =\left\langle h_{1} \otimes f_{1} \otimes S_{1}, g_{2} \otimes V\left(g_{1} \otimes w_{1} \otimes w_{2}\right)\right\rangle=\left\langle h_{1} \otimes S_{1}, g_{2} \otimes w_{1} \otimes \pi_{2}\left(f_{1} g_{1}\right)\left(w_{2}\right)\right\rangle \\
& =\left\langle T \pi_{1}\left(f_{2} g_{2}\right)\left(w_{1}\right), \pi_{2}\left(f_{1} g_{1}\right)\left(w_{2}\right)\right\rangle,
\end{aligned}
$$

and also

$$
\begin{aligned}
& \left\langle f_{1} \otimes f_{2} \otimes T, V_{13} U_{23}\left(g_{1} \otimes g_{2} \otimes w_{1} \otimes w_{2}\right)\right\rangle \\
& =\left\langle f_{2} \otimes V^{*}\left(f_{1} \otimes T\right),(\chi \otimes \mathrm{id} \otimes \mathrm{id})\left(g_{1} \otimes U\left(g_{2} \otimes w_{1} \otimes w_{2}\right)\right)\right\rangle \\
& =\left\langle h_{2} \otimes f_{2} \otimes S_{2}, g_{1} \otimes U\left(g_{2} \otimes w_{1} \otimes w_{2}\right)\right\rangle=\left\langle h_{2} \otimes S_{2}, g_{1} \otimes \pi_{1}\left(f_{2} g_{2}\right)\left(w_{1}\right) \otimes w_{2}\right\rangle \\
& =\left\langle T \pi_{1}\left(f_{2} g_{2}\right)\left(w_{1}\right), \pi_{2}\left(f_{1} g_{1}\right)\left(w_{2}\right)\right\rangle,
\end{aligned}
$$

which proves equality, as required.

Finally, let $x=x_{1} \otimes x_{2} \in E_{1} \widehat{\otimes}_{\alpha} E_{2}$ and let $\mu=\mu_{1} \otimes \mu_{2} \in\left(E_{1} \widehat{\otimes}_{\alpha} E_{2}\right)^{*}$. By Theorem [3.5, if $F \in L^{\infty}(X)$ is defined by

$$
\langle F, a\rangle=\langle\mu, \pi(a)(x)\rangle \quad\left(a \in L^{1}(X)\right)
$$

then $F \in \operatorname{WAP}\left(L^{\infty}(X)\right)$. Now, for $a \in L^{1}(X)$, pick $f, g \in L^{2}(X)$ with $f g=a$, so we see that

$$
\langle\mu, \pi(a)(x)\rangle=\left\langle f \otimes \mu_{1} \otimes \mu_{2}, W\left(g \otimes x_{1} \otimes x_{2}\right)\right\rangle=\left\langle f \otimes \mu_{1} \otimes \mu_{2}, U V\left(g \otimes x_{1} \otimes x_{2}\right)\right\rangle .
$$

Notice that for $k \in L^{2}(X), \lambda_{1} \in E_{1}^{*}$ and $\lambda_{2} \in E_{2}^{*}$,

$$
\begin{aligned}
\left\langle k \otimes \lambda_{1} \otimes \lambda_{2}, V\left(g \otimes x_{1} \otimes x_{2}\right)\right\rangle & =\left\langle\lambda_{1} \otimes \lambda_{2},\left(\mathrm{id} \otimes \pi_{2}(k g)\right)\left(x_{1} \otimes x_{2}\right)\right\rangle \\
& =\left\langle\lambda_{1}, x_{1}\right\rangle\left\langle\lambda_{2}, \pi_{2}(k g)\left(x_{2}\right)\right\rangle .
\end{aligned}
$$

For $T \in\left(E_{1} \widehat{\otimes}_{\alpha} E_{2}\right)^{*}=\mathcal{B}_{\alpha^{\prime}}\left(E_{1}, E_{2}^{*}\right)$, as $E \widehat{\otimes}_{\alpha} F$ is reflexive, we hence must have that

$$
\left\langle k \otimes T, V\left(g \otimes x_{1} \otimes x_{2}\right)\right\rangle=\left\langle T\left(x_{1}\right), \pi_{2}(k g)\left(x_{2}\right)\right\rangle .
$$


Define a map $\theta: L^{2}(X) \otimes E_{2} \rightarrow L^{2}(X) \otimes E_{1} \widehat{\otimes}_{\alpha} E_{2}$ by $\theta(k \otimes x)=\theta\left(k \otimes x_{1} \otimes x\right)$ for $k \in L^{2}(X)$ and $x \in E_{2}$ on elementary tensors. A simple calculation shows that $\theta$ extends to a contraction $L^{2}\left(X, E_{2}\right) \rightarrow L^{2}\left(X, E_{1} \widehat{\otimes}_{\alpha} E_{2}\right)$. Then, for $\tau \in L^{2}\left(X, E_{2}\right)$, we have that

$$
\left\langle k \otimes \lambda_{1} \otimes \lambda_{2}, \theta(\tau)\right\rangle=\left\langle\lambda_{1}, x_{1}\right\rangle\left\langle k \otimes \lambda_{2}, \tau\right\rangle,
$$

and so, similarly,

$$
\langle k \otimes T, \theta(\tau)\rangle=\left\langle k \otimes T\left(x_{1}\right), \tau\right\rangle .
$$

Let $\hat{V} \in L^{\infty}(X) \bar{\otimes} \mathcal{B}\left(E_{2}\right)$ be defined by $\pi_{2}$. Then

$$
\left\langle k \otimes T, V\left(g \otimes x_{1} \otimes x_{2}\right)\right\rangle=\left\langle k \otimes T\left(x_{1}\right), \hat{V}\left(g \otimes x_{2}\right)\right\rangle=\left\langle k \otimes T, \theta \hat{V}\left(g \otimes x_{2}\right)\right\rangle .
$$

Thus $V\left(g \otimes x_{1} \otimes x_{2}\right)$ is in the image of $\theta$, being equal to $\theta \hat{V}\left(g \otimes x_{2}\right)$.

Again, we use Sweedler notation, so by the previous paragraph, we may suppose that $V\left(g \otimes x_{1} \otimes x_{2}\right)=h \otimes x_{1} \otimes y_{2}$. Then, for $k \in L^{2}(X), \lambda_{1} \in E_{1}^{*}$ and $\lambda_{2} \in E_{2}^{*}$, we see that

$\left\langle k \otimes \lambda_{1} \otimes \lambda_{2}, h \otimes x_{1} \otimes y_{2}\right\rangle=\left\langle\lambda_{1} \otimes \lambda_{2},\left(\mathrm{id} \otimes \pi_{2}(k g)\right)\left(x_{1} \otimes x_{2}\right)\right\rangle=\left\langle\lambda_{1}, x_{1}\right\rangle\left\langle\lambda_{2}, \pi_{2}(k g)\left(x_{2}\right)\right\rangle$.

Hence we have that

$$
\left\langle k \otimes \lambda_{2}, h \otimes y_{2}\right\rangle=\left\langle\lambda_{2}, \pi_{2}(k g)\left(x_{2}\right)\right\rangle .
$$

Finally, we have that

$$
\begin{aligned}
\langle\mu, \pi(a)(x)\rangle & =\left\langle f \otimes \mu_{1} \otimes \mu_{2}, U\left(h \otimes y_{1} \otimes y_{2}\right)\right\rangle=\left\langle\mu_{1} \otimes \mu_{2},\left(\pi_{1}(f h) \otimes \mathrm{id}\right)\left(y_{1} \otimes y_{2}\right)\right\rangle \\
& =\left\langle\mu_{2}, y_{2}\right\rangle\left\langle F_{1}, f h\right\rangle=\left\langle F_{1} f \otimes \mu_{2}, h \otimes y_{2}\right\rangle \\
& =\left\langle\mu_{2}, \pi_{2}\left(\left(F_{1} f\right) g\right)\left(x_{2}\right)\right\rangle=\left\langle F_{2},\left(F_{1} f\right) g\right\rangle=\left\langle F_{2}, F_{1} f g\right\rangle=\left\langle F_{1} F_{2}, a\right\rangle .
\end{aligned}
$$

So we conclude that $F_{1} F_{2}=F \in \operatorname{WAP}\left(L^{1}(X)\right)$, showing that $\operatorname{WAP}\left(L^{1}(X)\right)$ is an algebra.

Corollary 3.8. Let $G$ be a locally compact group. Then $\operatorname{WAP}(M(G))$ is a $C^{*}$-subalgebra of $M(G)^{*}=C_{0}(G)^{* *}$.

\section{References}

[1] R. Aharoni, P. D. Saphar, 'On the reflexivity of the space $\pi_{p}(E, F)$ of $p$-absolutely summing operators, $1 \leq p<+\infty$ ', Bull. London Math. Soc. 25 (1993) 362-368.

[2] J. F. Berglund, H. D. Junghenn, P. Milnes, Analysis on semigroups; Function spaces, compactifications, representations, (John Wiley \& Sons, Inc., New York, 1989).

[3] H. G. Dales, Banach algebras and automatic continuity, (Clarendon Press, Oxford, 2000).

[4] H. G. Dales, A. T.-M. Lau, D. Strauss, 'The measure algebra and its second dual', preprint.

[5] W. J. Davis, T. Figiel, W. B. Johnson, A. Peeczyński, 'Factoring weakly compact operators', J. Functional Analysis 17 (1974) 311-327.

[6] M. DAws, 'Dual Banach algebras: representations and injectivity', Studia Math. 178 (2007) 231-275. 
[7] A. Defant, K. Floret, Tensor norms and operator ideals, (North-Holland, Amsterdam, 1993).

[8] J. Diestel, J. J. JR. Uhl, Vector measures, (American Mathematical Society, Providence, R.I., 1977).

[9] J. Duncan, A. ÜLger, 'Almost periodic functionals on Banach algebras', Rocky Mountain J. Math. 22 (1992) 837-848.

[10] E. Effros, Z.-J. Ruan, Operator spaces, (Oxford University Press, New York, 2000).

[11] N. Hindman, D. Strauss, Algebra in the Stone-Čech compactification, (Walter de Gruyter \& Co., Berlin, 1998).

[12] S. Kaijser, 'On Banach modules. I.', Math. Proc. Cambridge Philos. Soc. 90 (1981) 423-444.

[13] J. Kustermans, 'Locally compact quantum groups', in Quantum independent increment processes. I, 99-180, Lecture Notes in Math., 1865, (Springer, Berlin, 2005).

[14] J. Kustermans, L. Tuset, 'A survey of $C^{*}$-algebraic quantum groups. I', Irish Math. Soc. Bull. 43 (1999) 8-63.

[15] J. Kustermans, L. Tuset, 'A survey of $C^{*}$-algebraic quantum groups. II', Irish Math. Soc. Bull. 44 (2000) 6-54.

[16] A. T.-M. LAU, 'Analysis on a class of Banach algebras with applications to harmonic analysis on locally compact groups and semigroups', Fund. Math. 118 (1983) 161-175.

[17] T. W. PALmer, Banach algebras and the general theory of*-algebras, Vol 1, (Cambridge University Press, Cambridge, 1994).

[18] R. RYAN, Introduction to Tensor Products of Banach Spaces, (Springer-Verlag, London, 2002).

[19] M. TAKesaki, Theory of Operator Algebras I, (Springer-Verlag, New York-Heidelberg, 1979).

[20] A. ÜLGER, 'Continuity of weakly almost periodic functionals on $L^{1}(G)$ ', Quart. J. Math. Oxford Ser. (2) 37 (1986) 495-497.

[21] N. J. Young, 'Periodicity of functionals and representations of normed algebras on reflexive spaces.', Proc. Edinburgh Math. Soc. (2) 20 (1976/77) 99-120.

[22] J. C. S. Wong, 'Topologically stationary locally compact groups and amenability', Trans. Amer. Math. Soc. 144 (1969) 351-363.

Author's address: School of Mathematics,

University of Leeds,

Leeds LS2 9JT

United Kingdom

Email: matt.daws@cantab.net 